\title{
Species Level Identification of Yeast and Yeast Like Fungus for Prompt Infection Control Measures in Prevention of Outbreaks: With Special Reference to Candida auris in Pre-covid Era
}

\section{Gitali Bhagawati $^{1,2 *}$, Sarita Rani Jaiswal ${ }^{2,3}$, Ashutosh Bhardwaj, ${ }^{2,4}$, Navneet Sood ${ }^{2,5}$, Rekha Saji Kumar' ${ }^{1}$, Lincy TP ${ }^{2}$, Sania Paul' ${ }^{1}$, Mansi $^{1}$ and Suparno Chakrabarti ${ }^{2,3}$}

\author{
${ }^{1}$ Department of Microbiology, Dharamshila Narayana Superspeciality Hospital and \\ Research Centre, New Delhi, India \\ ${ }^{2}$ Hospital Infection Control Team, Dharamshila Narayana Superspeciality Hospital \\ and Research Centre, New Delhi, India \\ ${ }^{3}$ Department of Blood and Marrow Transplantation, Dharamshila Narayana \\ Superspeciality Hospital and Research Centre, New Delhi, India \\ ${ }^{4}$ Critical Care Medicine, Dharamshila Narayana Superspeciality Hospital and \\ Research Centre, New Delhi, India \\ ${ }^{5}$ Department of Pulmonology, Narayana Superspeciality Hospital and Research \\ Centre, New Delhi, India
}

*Corresponding Author: Gitali Bhagawati, Consultant and Head, Department of Microbiology and Infection Control, Dharmshila Narayana Superspeciality Hospital, Vasundhara Enclave, Delhi, India.
Received: June 03, 2021

Published: July 09, 2021

(C) All rights are reserved by Gitali

Bhagawati., et al.

\begin{abstract}
Background: Candida auris (C. auris) is emerging as a multi-drug resistant (MDR) strain of Candida amongst Non-albicans Candida (NAC) which poses a serious risk of nosocomial spread with high mortality rate.

Aim: Aim of the study is to give emphasis on species level identification of all yeast and yeast like fungus (YYLF) for the implementation of infection control practices (ICP) to prevent outbreak, with special reference to C. auris.

Methods: The study was done over a period of 12 months in a tertiary care hospital. YYLF isolated from primary culture were further sub-cultured on Sabouroud Dextrose Agar (SDA) and incubated at $25^{\circ} \mathrm{C}$ and $37^{\circ} \mathrm{C}$ for 72 hours. Identification and antifungal susceptibility was done using Vitek 2 Compact system 8.01 (bioMérieux, North Carolina/USA). The $1^{\text {st }}$ two isolates were confirmed by molecular method (D1-D2 sequencing). C. auris specific containment measures were implemented in the early part of the study. Clinical data and outcome were evaluated at end of the period.

Findings: Out of 2,487 non-duplicate samples processed over 12 months, YYLF were isolated from 209 (8.40\%) samples. Amongst the YYLF, predominant isolate was C. albicans $(109 / 209,52.15 \%)$, followed by $C$. tropicalis $(35 / 209,16.74 \%)$. Predominant source was urinary samples $(115 / 209,55 \%)$ followed by respiratory samples $(60 / 209,28 \%)$. C. auris was isolated in 7 non-duplicate samples $(7 / 209,3.35 \%)$. Out of these 7 cases, 5 had history of cancer $(72.42 \%)$. Measures for containment of $C$. auris were placed prior to the study and implemented at each time of its isolation. No nosocomial spread was detected during this study period.

Conclusion and Clinical Significance: Species level identification following proper quality control in microbiology laboratory along with appropriate sample collection can act as a primer in controlling outbreaks by YYLF. Pre-emptive implementation of hospital infection control (HIC) policies can help in controlling the MDR C.auris, even amongst immunocompromised patients.
\end{abstract}

Keywords: C. auris; Multi Drug Resistant; Non-albicans Candida; Infection Control Practices; Yeast and Yeast-like Fungus; Hospital Infection Control 


\section{Abbreviations}

C. auris: Candida auris; MDR: Multi Drug Resistant; NAC: Non-albicans Candida; ICP: Infection Control Practices; YYLF: Yeast and Yeast-Like Fungus; HIC: Hospital Infection Control; BAL: Bronchoalveolar Lavage; CSF: Cerebrospinal Fluid; CCA: Critical Care Areas; ICU: Intensive Care Unit; BMT: Bone Marrow Transplant; KTU: Kidney Transplant Unit; CLED: Cystine Lactose Electrolyte Deficient Agar; SDA: Sabouraud's Dextrose Agar; ATCC: American Type Culture Collection; WHO: World Health Organization; UWP: Universal Work Precautions; SUD: Single Use Device

\section{Introduction}

In 2009, a novel Candida species, Candida auris (C. auris) was isolated from external ear canal of a Japanese patient in Tokyo Metropolitan Geriatric Hospital [1]. In the same year, 15 cases of C. auris were isolated in South Korea from the ear canals of patients suffering from chronic otitis media [2]. During 2013 to 2014, two larger series of cases of persistent candidemia and deep-seated infections with high mortality rate were reported by multi drug resistant (MDR) strains of $C$. auris from 3 hospitals in India [3]. In Chicago, a hospital has been dealing with an outbreak of $C$. auris since March 2017, when its first case was confirmed. Since then, despite implementing all ICP, the proportion of residents testing positive has climbed to $71 \%$ [4].

Whole genome sequencing and epidemiological analysis proved that the MDR $C$. auris-related disease appeared independently on three continents (Indian subcontinent, Venezuela, and South Africa during 2012 to 2015) simultaneously [5]. One of the hypotheses predicts its emergence might have been linked to global warming effects on wet-lands where it prevailed as a plant saprophyte [6]. This study was done with the objectives to understand the prevalence and epidemiology of yeast and yeast like fungus (YYLF) with special reference to $C$. auris in a tertiary care hospital over 12 months along with the containment strategies by infection control measures.

\section{Materials and Methods}

Study design

The study was carried out in the department of microbiology in a tertiary care hospital in Delhi, India over a period of 12 months, from January 2018 to December 2018. The various clinical samples included in the study comprised of urine, blood, respiratory [sputum, bronchoalveolar lavage (BAL) etc.] and others [pus, wound swab, Cerebrospinal fluid (CSF), Ascitic fluid etc.] The samples were received from Critical care areas (CCA) [Intensive Care Unit (ICU), Bone marrow transplant (BMT) unit, Kidney Transplant unit (KTU) etc.], various indoor departments of the hospital and outdoors.
Inclusion criteria of the samples

- Urine: Wet mount showing pus cells $>=10 \mathrm{WBC} /$ cubic mm or pure growth in culture with colony count $>=10^{3} \mathrm{CFU} / \mathrm{ml}$ irrespective of pus cells in routine examination [8].

- Sputum: Samples showing Bartlett's score more than 1 [9].

- Bronchoalveolar lavage: Colony count $>=10^{4} \mathrm{CFU} / \mathrm{ml}$ in quantitative culture [10].

- Blood: Repeated isolation of same species in paired samples [10]. C. auris specific containment measures were implemented in the early part of the study and clinical data and outcome were evaluated at the end of this study.

\section{Microbiological testing}

All the samples were inoculated on routine culture media like blood agar, MacConkey agar, Cystine Lactose Electrolyte Deficient Agar (CLED). After growth in routine culture media, for cultural morphology, colonies were inoculated on two Sabouraud's Dextrose Agar (SDA) slants; one was incubated at room temperature and other at $37^{\circ} \mathrm{C}$ for $24-48$ hrs. The growth on the slope was processed for identification of the fungus.

From the isolated colony, Gram staining was done. Germ tube test was performed to differentiate Candida albicans from Nonalbicans Candida (NAC); slide culture and Dalmau plate [7] techniques were performed for speciation, using Corn meal agar. The final identification and antifungal susceptibility tests were performed by YST Identification cards and YST AST cards respectively using Vitek 2 Compact System 8.01 (bioMérieux, Inc. Durham, North Carolina/USA). Control strains used were: C. albicans ATCC (American Type Culture Collection) 14053, C. parapsilosis ATCC 22019 and C. krusei ATCC 14243.

Two of the $C$. auris isolates from Case1 and Case 2 were further confirmed by sequencing of internal transcribed spacer and D1/D2 regions in Reference lab, PGI, Chandigarh. This showed homology with the type $C$. auris reference strains. This helped us in inter-laboratory comparison. Once it was confirmed, all Vitek identified $C$. auris were considered as confirmed case and accordingly prompt infection control measures were implemented (Figure 1 and 2).

\section{Results}

Out of 2487 non-duplicate samples processed over 12 months, 209 samples grew YYLF (8.40\%). Majority of the culture positive samples for YYLF were from IPD (155/209, 74\%), followed by critical care areas and OPD (Figure 3). This is contributed to the immunocompromised status of the patients, mainly cancer patients admitted in the institute. Four $(4 / 7,57.14 \%)$ isolates of $C$. auris 


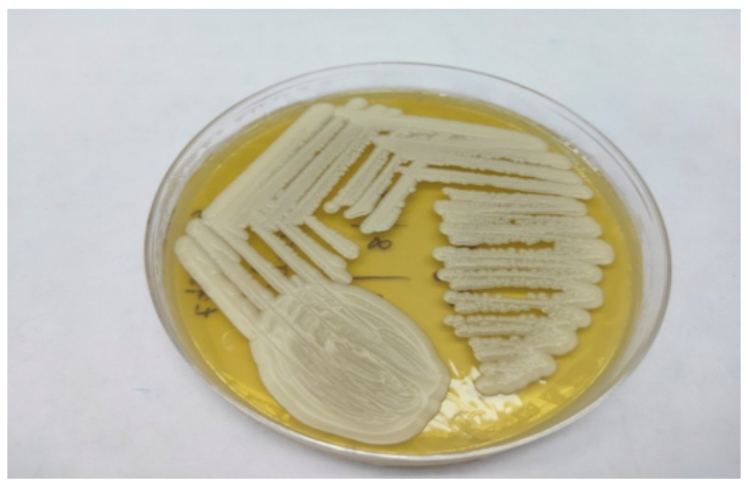

Figure 1: Colony morphology of C. auris in Sabouraud Dextrose Agar (SDA)

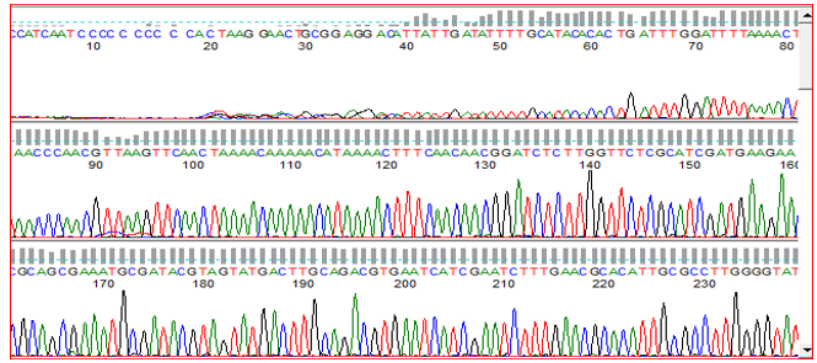

Figure 2: Sequencing of D1-D2 region of $C$. auris isolate 1.

were retrieved from indoor patients while three $(3 / 7,42.86 \%)$ from intensive care unit (Figure 3).

The predominant organism isolated was C. albicans $(109 / 209$, $52.15 \%)$, followed by C. tropicalis $(35 / 209,16.74 \%)$ and C. glabrata $(23 / 209,11 \%)$. The isolation rate of $C$. auris was 7 (7/209, $3.35 \%$ ) (Figure 3 and 4).

No C. haemulonii or $C$. duobushaemulonii was isolated which is supposed to be misidentified by Vitek 2 YST which was used for identification [11].

YYLF isolates were mostly retrieved from urinary samples $(115 / 209,55 \%)$ followed by respiratory samples $(60 / 209,28 \%)$ and blood $(20 / 209,9.57 \%)$. Five of the isolates of $C$. auris were from urinary samples $(5 / 7,71.43 \%)$ with colony count $>=100,000$ $\mathrm{CFU} / \mathrm{ml}$. One sample of Bronchoalveolar lavage fluid (BAL fluid) with colony count $>=10,000$ and the other sample of paired blood culture was found to be positive for $C$. auris (Figure 3 and table 1 ).

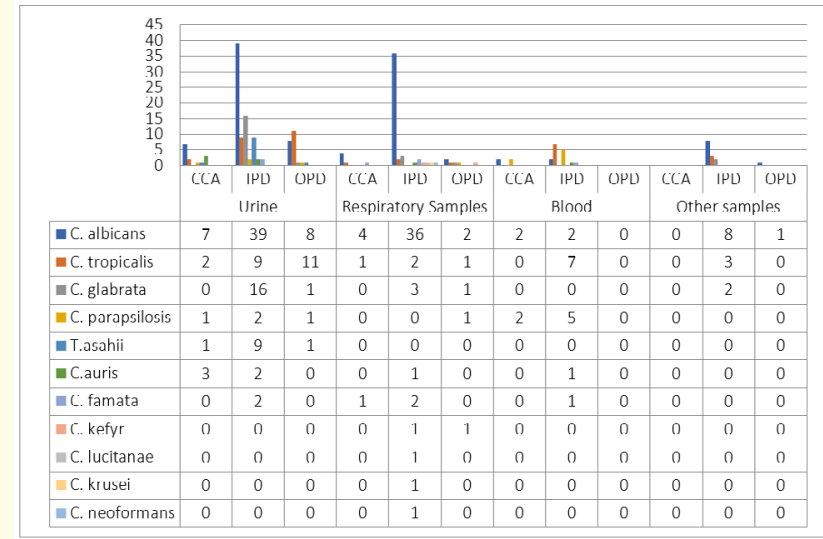

Figure 3: Distribution of Yeast and Yeast like fungus as per clinical samples and patient care area.

Note: Respiratory sample includes sputum, induced sputum, endotracheal aspirate, bronchoalveolar lavage etc. Other samples include pus, wound swab, stool, high vaginal swab etc. YYLF: Yeast and yeast like fungus, CCA: Critical care areas, IPD: Indoor patient Department, OPD: Outdoor patient Department.

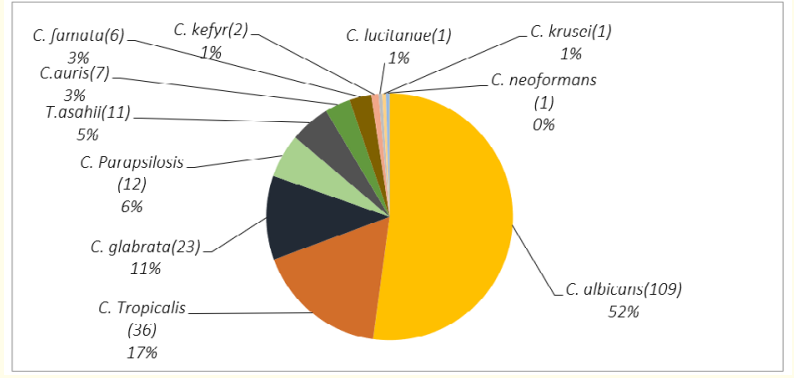

Figure 4: Distribution of Yeast and Yeast-like isolates from various samples.

On analysis of the cases, it was found that out of seven cases, five patients had history of cancer, one had history of chronic kidney disease (CKD) on dialysis and testicular abscess with type 2 diabetes mellitus (Type $2 \mathrm{DM}$ ) and the rest other had history of ruptured liver abscess with sepsis (Table 1).

Location of the patients were in different areas of the hospital, while their isolation was in different months of the year except two cases in the month of February and two in the month of December, 2018. Each case was identified promptly and infection control 
measures were taken. No nosocomial spread was detected during the study period. Out of 7 identified cases, one patient expired with mortality rate $14.28 \%$ (Table 1 ).

Out of seven isolates, six were resistant to fluconazole (MIC> $>32$ ) and amphotericin-B (MIC>=2). No isolate was found to be resistant to caspofungin or micafungin $[12,13]$ (Table 1$)$.

Policies of infection control implemented for each isolate of $C$. auris $[13,14]$ :

- To rule out colonization, surveillance samples in the form of nasal swab/swab from axilla were collected from nurses associated with the infected patients. A total 16 swabs were collected and processed in Microbiology laboratory for culture. However, no swab was found to be positive for $C$. auris.
- Environmental swabs were collected from door handles, bed rails, cannula hubs etc. in each case of $C$. auris isolate. Bed rail of one case (Case 3) (Table 1) was found to be positive for $C$. auris. Stringent cleaning protocol with hydrogen peroxide and sodium hypochlorite with proper concentration and contact period as per manufacturers' recommendation was followed. Due to the fixed cleaning protocols, no swab was found to be positive for $C$. auris.

- Revision of Isolation policy: The list of contact isolation policy of the hospital has been extended including $C$. auris.

- Two dedicated rooms with dedicated staff, Bio-medical waste dust bins and separate bed pans were allocated for patients positive for $C$. auris.

- Signage of Contact isolation was displayed outside the room.

\begin{tabular}{|c|c|c|c|c|c|c|c|c|}
\hline & $\begin{array}{l}\text { Patient's } \\
\text { Details }\end{array}$ & Patient 1 & Patient 2 & Patient 3 & Patient 4 & Patient 5 & Patient 6 & Patient 7 \\
\hline 1 & Age(years) & 65 & 67 & 66 & 61 & 65 & 26 & 48 \\
\hline 2 & Gender & Male & Male & Female & Male & Female & Male & Female \\
\hline 3 & $\begin{array}{c}\text { Area of } \\
\text { admission }\end{array}$ & $2^{\text {nd }}$ floor & $1^{\text {st }}$ floor & ICU & $2^{\text {nd }}$ floor & ICU & ICU & $2^{\text {nd }}$ floor \\
\hline 4 & Month (2018) & Feb & Feb & April & June & Oct & Dec & Dec \\
\hline 5 & $\begin{array}{c}\text { Sample positive } \\
\text { for } C \text {. auris }\end{array}$ & BAL & Urine & Urine & Blood & Urine & Urine & Urine \\
\hline 6 & Colony count & $>=10,000$ & $>=100,000$ & $>=10,000$ & NA & $>=100,000$ & $>=100,000$ & $>=100,000$ \\
\hline 7 & $\begin{array}{l}\text { Predisposing } \\
\text { factor }\end{array}$ & $\begin{array}{c}\text { Type } 2 \mathrm{DM} \\
\text { with lower } \\
\text { respiratory } \\
\text { tract infection }\end{array}$ & \begin{tabular}{|c|} 
CKD (StageIV), \\
Hypocontrac- \\
tile bladder, \\
HTN, \\
Type 2 DM, \\
Hypothyroid- \\
ism, UTI \\
\end{tabular} & $\begin{array}{c}\text { On and off hospital } \\
\text { admission since } \\
\text { Oct, 2017; Patient } \\
\text { was on ventilator } \\
\text { and urinary cath- } \\
\text { eter }\end{array}$ & \begin{tabular}{|} 
Patient was on \\
chemotherapy \\
since April, \\
2018
\end{tabular} & $\begin{array}{l}\text { T2DM, HTN } \\
\text { and parapa- } \\
\text { resis }\end{array}$ & Nil & $\begin{array}{c}\text { Pituitary } \\
\text { adenoma and } \\
\text { aneurysm } \\
\text { diagnosed in } \\
1997\end{array}$ \\
\hline 8 & $\begin{array}{l}\text { History of sur- } \\
\text { gery }\end{array}$ & \begin{tabular}{|c|} 
Decompessive \\
craniotomy \\
with tumour \\
excision in \\
2017
\end{tabular} & $\begin{array}{l}\text { Orchidectomy } \\
\text { (March, 2015) }\end{array}$ & \begin{tabular}{|c} 
Post-operative \\
case of TAH \\
with B/L RPLND, \\
omentectomy (Oct, \\
2017); on CT
\end{tabular} & Nil & Nil & $\begin{array}{c}\text { Craniotomy and } \\
\text { Tumour decom- } \\
\text { pression }\end{array}$ & $\begin{array}{l}\text { Craniotomy } \\
\text { and Clipping } \\
\text { of Aneurysm } \\
\text { done in } 2018\end{array}$ \\
\hline 9 & Diagnosis & $\begin{array}{c}\text { Glioblastoma } \\
\text { multiforme }\end{array}$ & $\begin{array}{c}\text { CKD(StageIV) } \\
\text { with recurrent } \\
\text { UTI }\end{array}$ & $\begin{array}{c}\text { Recurrent CA Right } \\
\text { Ovary with ileal } \\
\text { perforation with } \\
\text { sepsis }\end{array}$ & $\begin{array}{l}\text { Carcinoma } \\
\text { Lung }\end{array}$ & \begin{tabular}{|c|} 
Ruptured \\
Liver abcess \\
with B/L \\
pneumonia \\
with sepsis \\
with recurrent \\
UTI \\
\end{tabular} & $\begin{array}{l}\text { Esthesioneuro- } \\
\text { blastoma }\end{array}$ & $\begin{array}{l}\text { Subarach- } \\
\text { noid hemor- } \\
\text { rhage (SAH) }\end{array}$ \\
\hline 10 & $\begin{array}{c}\text { No. of Hospital } \\
\text { days }\end{array}$ & 24 & 10 & $\begin{array}{l}\text { Day care treatment } \\
\text { for Chemotherapy }\end{array}$ & 20 & 37 & 12 & 35 \\
\hline 11 & $\begin{array}{c}\text { H/O use of } \\
\text { Broad spectrum } \\
\text { antibiotic } \\
\text { antibiotics }\end{array}$ & $\begin{array}{c}\text { Colistin, } \\
\text { Linezolid, } \\
\text { Tigecycline }\end{array}$ & $\begin{array}{l}\text { Meropenem, } \\
\text { Teicoplanin }\end{array}$ & $\begin{array}{l}\text { Meropenem, } \\
\text { Teicoplanin }\end{array}$ & $\begin{array}{l}\text { Colistin, } \\
\text { Fosfomycin }\end{array}$ & $\begin{array}{l}\text { Polymyxin B, } \\
\text { Fosfomycin, } \\
\text { Minocycline, } \\
\text { Linezolid }\end{array}$ & $\begin{array}{c}\text { Colistin, } \\
\text { Vncomycin }\end{array}$ & $\begin{array}{c}\text { Colistin, } \\
\text { Vancomycin, } \\
\text { Linezolid }\end{array}$ \\
\hline
\end{tabular}


Species Level Identification of Yeast and Yeast Like Fungus for Prompt Infection Control Measures in Prevention of Outbreaks: With Special Reference to Candida auris in Pre-covid Era

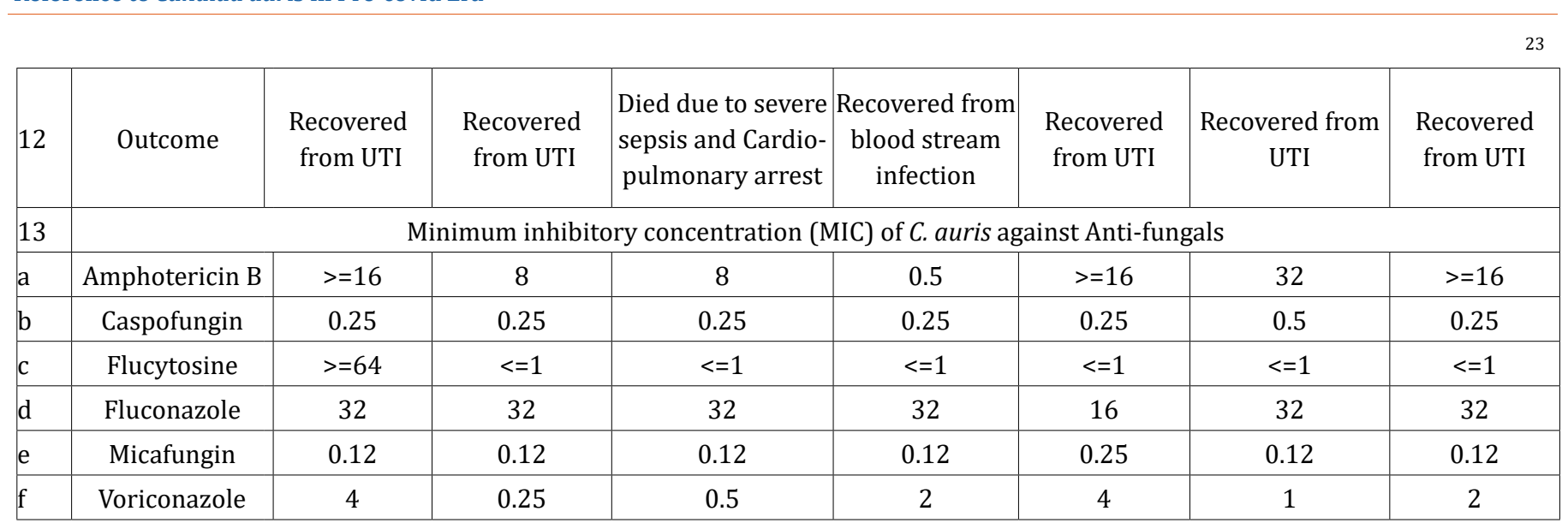

Table 1: Details of cases infected with C. auris.

Similarly, in ICU also, two rooms were allocated for such positive patients for contact isolation:

- Stringent policy on hand hygiene: 5 moments of Hand Hygiene and Steps of Hand hygiene recommended by World health organization (WHO) was followed stringently. Audit of Hand hygiene was done regularly using hand hygiene data sheet provided by WHO. One hourly alarm for hand washing is being followed in ICU area. This is followed by all staff including doctors in ICU.

- Universal Work precautions (UWP): Apart from hand hygiene, other measures of UWP were followed like wearing gowns, gloves, proper sterilization and disinfection of instruments etc. Mostly Single use device (SUD) preferred for patient care; high level disinfection by ethylene oxide or by autoclave was done for other items.

\section{Discussion}

In the year 2018, total 209 non-duplicate samples were found to be positive for YYLF in various samples with positivity rate $2.34 \%$ (209/8918). Amongst the YYLF, C. albicans was the predominant yeast (52\%) and the rest were NAC (48\%). Among all NAC, C. tropicalis predominated with $17 \%$, followed by C. glabrata, $11 \%$; rate of isolation of $C$. auris was only $3 \%$ despite a notorious for nosocomial spread.

Similar data was found in a study done from National Hospital Discharge Survey (NHDS), United States which revealed rate of invasive Candidiasis was predominated by $C$. albicans ranging from $73.3 \%$ in 1997 to $62.3 \%$ in 2003. No Candida auris was isolated during these periods; however unidentified spp. of Candida ranged from $3.9 \%$ in 1997 to $4.9 \%$ in 2003 [15].

Candidemia was seen in $9.57 \%$ (20/209) cases in our study. NAC candidemia $80 \%(16 / 20)$ predominated; $35 \%$ of which was contributed by $C$. tropicalis and C. parapsilosis each. C. auris also contributed to candidemia in one indoor case (5\%) [Table 1]. Similar finding was detected from a 13-year long study on candidemia from a tertiary care hospital in Thailand showing prevalence rate of $6.14 \%$ for Candida species. NAC candidemia 134 (57.1\%) predominated over C. albicans 101 (42.9\%) [16]. In another study, prevalence of candidemia in infants was found to be $1.4 \%$. NAC was responsible for $56.5 \%$ of neonatal candidemia; C. glabrata $33.3 \%$ followed by $C$. tropicalis $20.3 \%$ [17]. No C. auris or unidentified Candida spp. was mentioned in these studies $[16,17]$.

In Kenya, C. auris was considered as the most common pathogen responsible for candidemias in a reference hospital accounting for 45 (38\%) episodes over a period of 3 years (2010 to 2013) [18]. During 2009-2011, 12 cases of candidaemia by C. auris were identified 2 hospitals (a tertiary care general hospital and a pediatric center) in Delhi, India [3]. Similarly, a study of 27 ICUs across India found $5.7 \%$ of C. auris candidemia from April 2011 to September 2012 [19].

Urinary tract infection (UTI) was predominated by C. albicans, $25.83 \%$ (54/209) followed by C. tropicalis $10.52 \%$ (22/209) and $C$. glabrata $8.13 \%(17 / 209)$. C. auris contributed to $2.39 \%(5 / 209)$ of UTI in indoor patients. In one study from Pakistan revealed that 193 isolates of $C$. auris among 92 patients; out of which 38 had candideamia and 19 patients had UTI [20]. Globally, few cases of UTI have been reported by $C$. auris. This might be because of under-reporting of the isolates due to lack of proper microbiology laboratory all over the globe especially in lower to middle income group countries. Secondly, lack of amenities for species level identification in most of the other laboratories. Third, under reporting can be a cause of false reporting of the cases as many different automated systems falsely identify it as various different Candida spp. like Candida haemulonii, Candida duobushaemulonii by Vitek 2 
Species Level Identification of Yeast and Yeast Like Fungus for Prompt Infection Control Measures in Prevention of Outbreaks: With Special Reference to Candida auris in Pre-covid Era

Compact; Candida haemulonii, Candida catenulate by BD Phoenix yeast identification system; Microscan misidentifies it as Candida famata, Candida guilliermondii, Candida lusitaniae and Candida parapsilosis [14].

C. auris can spread in healthcare settings through contact with contaminated environmental surfaces or equipment, or from person to person $[13,14]$. However, due to stringent IPC, no outbreak was seen in our hospital. Cluster of seven cases were seen in different months of the year (except 2 cases in February and 2 cases in December, 2018) at different locations amongst these immunocompromised patients (Table 1).

Out of seven isolates, six were resistant to fluconazole (86\%) which is similar to the findings of other studies [93\% [5] and 100\% [20]]. In our study, amphotericin-B resistance was $86 \%$ which was not in concordance with the findings of other studies [35\% [5] and $7.9 \%$ [20]]. No isolate was found to be resistant to echinocandins which is dissimilar to the result found by echinocandins [(5-10\%) [18] and 7\% [5]] (Table 1).

\section{Conclusion}

With the use of broad spectrum antibiotics, NAC has emerged as an important cause of infection in hospitalized patients with associated risk factors. Species level identification following proper quality control of microbiology laboratory with proper sample collection can act as a primer for prevention of outbreak by YYLF. Continuous monitoring and surveillance is necessary for all YYFL for the changing epidemiological pattern. $C$. auris is emerging MDR yeast which spreads readily between patients in healthcare setups. In our set-up, although isolation rate of $C$. auris infected patients were less in number, it was significant because of immediate and efficient containment with no nosocomial spread among the immunocompromised patients. Therefore, it is now essential that microbiology laboratories should identify clinical isolates of Candida upto the species level along with the antifungal susceptibility pattern. Stringent IPC focusing on source isolation, hand hygiene and disinfection are the most effective measure to prevent nosocomial spread by $C$. auris.

\section{Acknowledgements}

The authors acknowledge Dr. Arunaloke Chakrabarti, Prof. and Head, National Culture Collection for Pathogenic Fungi, Department of Medical Microbiology, PGIMER, Chandigarh, India, for reconfirming the isolates by sequencing.

\section{Conflict of Interest}

All authors report no conflicts of interest relevant to this article.

\section{Bibliography}

1. Satoh K., et al. "Candida auris sp. nov., a novel ascomycetous yeast isolated from the external ear canal of an inpatient in a Japanese hospital". Microbiology Immunology 53 (2009): 4144.

2. Kim MN., et al. "Candida haemulonii and closely related species at 5 university hospitals in Korea: identification, antifungal susceptibility, and clinical features". Clinical Infectious Diseases 48 (2009): 57-61.

3. Chowdhary A., et al. "New clonal strain of Candida auris, Delhi, India”. Emergency Infectious Diseases 19 (2013): 1670-1673.

4. Dal Mas C., et al. "Effects of the Natural Peptide Crotamine from a South American Rattlesnake on Candida auris, an Emergent Multidrug Antifungal Resistant Human Pathogen". Biomolecules 9.6 (2019): 205.

5. Lockhart SR., et al. "Simultaneous emergence of multidrugresistant Candida auris on 3 continents confirmed by wholegenome sequencing and epidemiological analyses". Clinical Infectious Diseases 64 (2017): 134-140.

6. Casadevall A., et al. "On the Emergence of Candida auris: Climate Change, Azoles, Swamps, and Birds". American Society for Microbiology 10.4 (2019): 01397-1419.

7. LJR Milne. Fungi. Mackie and McCartney Practical Medical Microbiology. J.G. Colle, A.G. Fraser, B.P. Marmion, A. Simmons Elsevier 14 (2008): 695-717

8. "Bailey and Scott's Diagnostic Microbiology". Betty A. Eorbes, Daniel F.Sahm, Alice S. Weissfeld. Infections of Urinary tract. Mosby Elsevier 12 (2007): 842-855

9. Wong LK., et al. "Comparison of six different criteria for judging the acceptability of sputum specimens". Journal of Clinical Microbiology 16. 4 (1982): 627-631.

10. JG Collee and W Marr. "Culture of Bacteria". Mackie and McCartney Practical medical Microbiology. Elsevier 14 (2008): 113-129.

11. https:/www.cdc.gov>fungal $>$ candida auris> Identification of Candida auris.

12. https:/www.cdc.gov>fungal $>$ candida auris $>$ antifungal susceptibility pattern and interpretation.

13. https:/www.icmr.nic.in>sites>default>files>guidelines>candi da auris. 
14. https://www.cdc.gov>fungal>candida auris>laboratories and Health Professionals> Infection Prevention and Control.

15. Pfaller MA and Diekema DJ. "Epidemiology of invasive candidiasis: a persistent public health problem". Clinical Microbiology Review 20.1 (2007): 133-163.

16. Tritipwanit K., et al. "Epidemiology of candidemia at King Chulalongkorn Memorial Hospital, Thailand". Journal of Infectious Diseases and Antimicrobial Agents 22 (2005): 59-69.

17. Fu J., et al. "Epidemiology of Candida albicans and non-C.albicans of neonatal candidemia at a tertiary care hospital in western China". BMC Infectious Diseases 17.1 (2017): 329.

18. Cortegiani A., et al. "Epidemiology, clinical characteristics, resistance, and treatment of infections by Candida auris". Journal of Intensive Care 19.6 (2018): 69.

19. Chakrabarti A., et al. "Incidence, characteristics and outcome of ICU-acquired candidemia in India”. Intensive Care Medicine 41.2 (2015): 285-295.

20. Sayeed MA., et al. "Clinical spectrum and factors impacting outcome of Candida auris: a single center study from Pakistan". BMC Infectious Diseases 19 (2019): 384.

Volume 4 Issue 8 August 2021

(C) All rights are reserved by Gitali Bhagawati., et al. 